ISSN: $1130-3743$ - e-ISSN: 2386-5660

DOI: http://dx.doi.org/10.14201/teoredu2017291273294

\title{
AMENAZAS Y RIESGOS CLIMÁTICOS EN POBLACIONES VULNERABLES. EL PAPEL DE LA EDUCACIÓN EN LA RESILIENCIA COMUNITARIA
}

\author{
Threats and climate risks into vulnerable populations. \\ The role of education in the community resilience
}

\section{Menaces et risques climatiques dans les populations vulnérables. Le rôle de l'éducation sur la résilience communautaire}

Edgar Javier GONZÁLEZ-GAUDIANO y Ana Lucía MALDONADO-GONZÁLEZ Universidad Veracruzana. Instituto de Investigaciones en Educación.

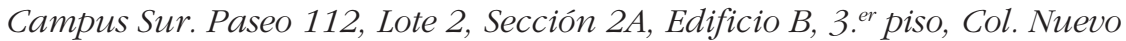
Xalapa91097Xalapa,Ver.México.edgagonzalez@uv.mx; anmaldonado@uv.mx

Fecha de recepción: enero de 2017

Fecha de aceptación: marzo de 2017

RESUMEN

Los inéditos desafíos del mundo contemporáneo obligan a la educación a replantear sus temas convencionales. La educación ambiental no es la excepción. La magnitud y complejidad de problemas ambientales globales como el cambio climático, la acidificación oceánica y la pérdida de la biodiversidad planetaria han relegado los asuntos que habían ocupado la atención pedagógica en este campo durante décadas. En este artículo se presentan los resultados iniciales de un estudio dirigido a valorar la percepción del riesgo y de vulnerabilidad de comunidades que de manera recurrente son afectadas por fenómenos hidrometeorológicos extremos. Los hallazgos han de fungir como punto de partida para el diseño de programas educativos orientados a fortalecer la resiliencia comunitaria. Partimos del supuesto 
de que, al basarnos en factores sociocognitivos que determinan las disposiciones a actuar por parte de poblaciones bajo presiones similares, podemos encontrar claves que nos permitan interpretar sus reacciones frente a situaciones difíciles. Esto considerando su experiencia previa y sus singularidades en la adaptación al cambio climático, en el aprendizaje social en momentos extremos y en la identificación de sus fortalezas y debilidades.

Palabras clave: riesgo; vulnerabilidad; resiliencia comunitaria; educación ambiental; Veracruz.

\section{SUMMARY}

Nowadays, challenges in the contemporary world lead to the education to propose its current themes. Environmental education is not an exception. The magnitude and complexity of global environmental problems such as the climate change, the ocean acidification and the loss of the biodiversity have generated issues that had attracted pedagogical attention for decades. This article presents the early results of a study aimed at assessing the perception of risk and vulnerability of communities that frequently are affected by extreme hydrometeorological phenomena. These findings could be a starting point for the design of educational programs aimed at strengthening community resilience. We start from the assumption based on sociocognitive factors that determine the dispositions in order to the populations can act under similar circumstances, we can find key elements that allow us to infer their reactions to difficult situations. This considering their previous experience and their singularities in the adaptation to climate change, in the social learning in extreme situations and in the identification of their strengths and weaknesses.

Key words: risk; vulnerability; community resilience; environmental education; Veracruz.

\section{SOMMAIRE}

Aujourd'hui, les défis au monde contemporain incitent à l'éducation à renouveler leurs actuels sujets d'etude. Évidemment, l'éducation environnementale ne fait pas l'exception. Tant la magnitude autant que la complexité des problèmes environnementaux mondiaux tels que le changement climatique, l'acidification des océans et la perte de la biodiversité mondiale ont oubli les questions qui avaient attiré l'attention pédagogique pendant des décennies. Dans cet article, on aborde les premiers résultats d'une étude visant à évaluer la perception du risque et de la vulnérabilité des communautés qui se sont vues affectées par des phénomènes météorologiques extrêmes. Les résultats donneront des pistes et des bases estratégiques afin de concevoir des programmes éducatifs visant à renforcer la résilience des communautés. D'après nous, à partir des facteurs socio-cognitifs qui déterminent les dispositions à agir de la part des populations sous des conditions similaires, nous pouvons trouver des indices qui nous permettent d'interpréter leurs réactions à des 
situations défavorables, compte tenu de leur expérience et de leurs singularités dans l'adaptation au changement climatique, dans l'apprentissage social sur des situations extrêmes et dans l'identification de leurs forces et de leurs faiblesses.

Mots clés: risque; vulnérabilité; résilience communautaire; éducation à l'environnement; Veracruz.

\section{INTRODUCCIÓN}

Los riesgos ambientales constituyen una de las principales limitantes de la sustentabilidad del desarrollo humano. En el caso del cambio climático, los riesgos se encuentran asociados a amenazas globales potencialmente destructivas y al grado de vulnerabilidad y exposición de la población. Experiencias recientes en países en desarrollo muestran que hasta eventos de pequeña escala pueden convertirse en grandes desastres para poblaciones expuestas en función de la adscripción geográfica; la desigualdad social relacionada específicamente al tipo de construcción de las viviendas y su localización; el nivel de organización comunitaria y la calidad de la gestión gubernamental, entre otros factores. Por su parte, Beck afirma que los riesgos son reales por sus efectos pero se constituyen a partir de cómo son percibidos socialmente. Un mismo riesgo es percibido y manejado de forma diferente en cada contexto. "El concepto de riesgo delimita, por tanto, un peculiar estado intermedio entre seguridad y destrucción, donde la percepción de riesgos amenazantes determina pensamiento y acción" (Beck, 2000, 10). El IPCC (2014) explica que la diferencia entre vulnerabilidad y exposición deriva de factores distintos al clima; en esto influyen desigualdades multidimensionales producidas por procesos de desarrollo dispares que ocasionan riesgos diferenciados, según las características de la población expuesta. Así, el riesgo es producto de la radical articulación de la vulnerabilidad, la exposición y la amenaza. En ese orden.

Denominamos resiliencia social a la capacidad comunitaria de encarar una adversidad, de salir de ella conservando su estabilidad organizativa, de desarrollar la capacidad social de aprender de la misma y de gestionar el cambio de algunas condiciones de riesgo y vulnerabilidad. La resiliencia constituirá una característica cada vez más trascendente en la medida en que se profundicen las consecuencias del conjunto de fenómenos sociales, económicos, ambientales y políticos que distinguen el momento de crisis epocal (Moore, 2011) por el que transitamos, al cual se ha denominado sociedad global del riesgo (Beck, 2008). Por lo mismo, es preciso como científicos sociales involucrarnos en su estudio de manera que se eleve como un propósito primario de procesos educativos y de cambio social, sobre todo cuando trabajamos con poblaciones vulnerables. Como lo apunta Sauvé (2010), hay que formar científicos que se comprometan en proyectos de resiliencia socioambiental mediante actividades creativas para reinventar el mundo. Agrega Sauvé que la educación ambiental invita a un proceso transformativo en lo concerniente a nuestra relación individual y colectiva con el medio ambiente, a fin 
de reconstruir la red de relaciones entre sociedad y ambiente. Ello para buscar el despliegue de capacidades para tomar decisiones, resolver problemas y gestionar nuestro ambiente con una visión del mundo y de nosotros en el mundo. Tales habilidades son particularmente necesarias para avanzar hacia la resiliencia social.

Las poblaciones que habitan en zonas costeras tropicales tienen una creciente vulnerabilidad frente a fenómenos meteorológicos extremos, cada vez más intensos y frecuentes, que son asociados al cambio climático con o sin fundamento. Adger (1999) define la vulnerabilidad como la exposición de grupos o individuos al estrés de los medios de vida que derivan de impactos provocados por diversos fenómenos. Uno de ellos es el cambio climático. En efecto, este fenómeno es epicentro de variados enfoques y discusiones; se trata de un tema recurrente al que la ciudadanía y diversos actores sociales refieren como causa y efecto de problemáticas ambientales que afectan a la población mundial de manera diferenciada, pero con mayor fuerza a las zonas costeras y los países tropicales e insulares. Empero, estudios en poblaciones diversas reportan un bajo reconocimiento de las implicaciones actuales y futuras del cambio climático en sus vidas (Norgaard y Rudy, 2008), al que se adiciona un alto el grado de desinterés, desconocimiento y mala interpretación de este fenómeno (Nisbet y Myers, 2007; Brachin, 2003; Dunlap, 1998).

La indiferencia social y política sobre el cambio climático genera que no se induzcan los cambios necesarios en los patrones de producción y consumo para mitigar la emisión de gases de efecto invernadero, sobre todo en un momento de crisis económica y financiera persistente. Asimismo, que no se adopten las medidas apropiadas para adaptarse a los cambios palmarios que están ocurriendo a todo nivel, aunque con mayores grados de afectación en las poblaciones vulnerables. La desigualdad social y la injusticia ambiental existentes se manifiestan con toda claridad en la carencia de políticas efectivas de respuesta ante el fenómeno.

La vulnerabilidad ha sido abordada por diversos autores. Fernández et al. (2016) señalan que hay cuatro dimensiones a considerar en la vulnerabilidad: 1) la dimensión física, la cual involucra el potencial de peligro por el impacto que reciben las infraestructuras; 2) la dimensión económica, que colisiona los activos económicos del lugar afectado; 3) la dimensión social, que concierne a individuos y comunidades humanas; y 4) la dimensión ambiental, referida a los impactos al medio ambiente y la capacidad de los ecosistemas para enfrentarlos.

Por su parte, Wilches-Chaux (1993) concibe la vulnerabilidad como la incapacidad de una comunidad para enfrentar un determinado riesgo que deriva en desastre. Analiza la vulnerabilidad desde distintos ángulos que pueden relacionarse entre sí, ya que la frontera entre unos y otros es muy sutil. De este modo, propone clasificarla en vulnerabilidad natural, física, económica, social, política, técnica, ideológica, cultural, educativa, ecológica e institucional. Anderson y Woodrow (1989) agrupan varios de los tipos de Wilches-Chaux y los condensan en sólo tres clases: 1) vulnerabilidad física (condiciones de clima, localización, suelo, niveles de la actividad productiva, salud, características de la vivienda, etc.); 2) vulnerabilidad social (sistema político y aparato institucional, demografía, procesos de decisión 
formales e informales de organización de actividades socioeconómicas); y 3) vulnerabilidad motivacional-actitudinal (la forma en que se ven a sí mismos los grupos sociales y sus capacidades para manejar su propio entorno). Ajustando el concepto a la situación climática, Neri y Aldunce (2008) afirman que la vulnerabilidad de una población difiere según su exposición a variaciones climáticas, su sensibilidad al grado de afectación y su capacidad de adaptación.

Como podemos inferir, al estar la vulnerabilidad relacionada con condiciones físicas, económicas, políticas y sociales de la población, es por lo que un mismo fenómeno tiene distintos grados de afectación, dependiendo de los rasgos del sitio y de quienes en él habitan. De ahí que el concepto de vulnerabilidad remita a condiciones particulares que producen efectos sinérgicos que se potencian entre sí. En otras palabras, se trata de una peculiaridad situada, desigual y acumulativa. A mayor desigualdad social, de exposición a fenómenos, así como menor organización social y política, la vulnerabilidad se incrementa por afectar las capacidades sociales adaptativas. Debido a la sinergia de los factores que determinan la vulnerabilidad, podemos reducirla si auspiciamos la organización social, mejorando la percepción social y la gestión participativa de los riesgos existentes, el aprendizaje social de la experiencia previa, la memoria colectiva de los acontecimientos vividos, así como la identificación de las características comunitarias que pueden catalizarse para fortalecer los procesos de resiliencia con base en derechos ciudadanos. Es decir, el problema de la vulnerabilidad y el riesgo puede asumirse como un reto educativo que apunta a contribuir a la constitución de lo que denominamos una ciudadanía climática. Dicha ciudadanía implica derechos y obligaciones políticas sociales para intervenir activamente en la construcción de estilos de vida orientados a frenar el cambio climático, con base en las responsabilidades que corresponden a los distintos agentes que constituyen la estructura social.

Por todo lo anterior, el riesgo y la vulnerabilidad son construcciones intersubjetivas con base en creencias y conocimientos de sentido común, compartidos por sujetos que viven los mismos acontecimientos. Halbwachs (2004) afirma que cada grupo se divide y se afianza en el tiempo y en el espacio y en este marco desarrolla la memoria colectiva original que permite recordar acontecimientos relevantes para el grupo. Entre menor sea el tamaño del grupo mayor será el interés colectivo otorgado a un evento. De este modo, la percepción del riesgo es particular en cada sociedad según las amenazas y la primacía social y política que se le asigna; no se trata de una percepción definitiva y clausurada, sino de una cuya significación es relacional, abierta, incompleta, provisional y precaria, regida por el espacio y el tiempo en que vive la población. De ahí la diferencia entre el riesgo real (objetivo) y el riesgo percibido (subjetivo). El riesgo real es estimado mediante procesos estadísticos que miden la probabilidad de impacto de cierta amenaza según la vulnerabilidad física y social. El riesgo percibido refiere a la forma en que el sujeto significa un fenómeno determinado, el que por ende varía según el espacio y el tiempo (Ávila Flores y González-Gaudiano, 2014). 
Podemos entonces decir que la causa principal en el aumento del riesgo social no proviene precisamente de la amenaza a enfrentar, sea ésta derivada del cambio climático o no, sino que proviene de la vulnerabilidad de la población y cómo ésta se incrementa progresivamente en la vida cotidiana como un proceso naturalizado, normalizado, hasta que llega un nuevo desastre. El riesgo es una construcción social en íntima relación con la vulnerabilidad. Eso convierte a la vulnerabilidad en la placenta en la que se gestan los desastres. La vulnerabilidad y la resiliencia son características inversamente proporcionales; es decir, mientras más resiliencia menos vulnerabilidad y viceversa.

García Acosta (2005) sugiere que para enfrentar los diversos componentes y dimensiones de la vulnerabilidad que caracterizan a la sociedad actual es necesario transitar de la administración del desastre a la gestión del riesgo, encarando así todo aquello que limita los medios de vida, la autoprotección y una intervención social apropiada. Esto es lo que puede generar condiciones que favorezcan la resiliencia comunitaria (Suárez Ojeda, 2001) al desarrollar la capacidad de aprender a partir de un desastre que ha afectado a una comunidad entera, así como al propiciar la movilización de competencias solidarias para reponerse de los daños y recuperar en la medida de lo posible la dinámica cotidiana de sus vidas.

Ahí radica nuestro especial interés en tanto autores de este artículo, por mostrar la significación del riesgo desde la perspectiva de los actores y de la relevancia que éstos otorgan a los distintos problemas que son capaces de identificar en cada una de las tres localidades estudiadas. Mostraremos el riesgo percibido de la población que varía según la visión de cada actor, aunque se trate de una misma localidad, lo cual explica al menos en parte la vulnerabilidad diferencial en la que viven las personas en determinado sitio, incluso contando con niveles socioeconómicos y culturales similares.

A partir de la asunción de que la amenaza no es la causa principal del riesgo, sino la vulnerabilidad en la que se encuentra una población expuesta (Viand y González, 2012; Briones, 2010), nos interesa mostrar aquí los aspectos que los distintos grupos de población identifican como causas de su vulnerabilidad. Como ya hemos dicho arriba, consideramos que ésta es una condición necesaria para contribuir a aminorar su vulnerabilidad. Es preciso que, desde distintas plataformas, incluida la educación y específicamente desde la educación ambiental, se colabore con las poblaciones en la definición y puesta en marcha de estrategias que contribuyan efectivamente a mitigar sus condiciones de vulnerabilidad a los embates del cambio climático, mediante acciones de adaptación, gestión de riesgos y resiliencia comunitaria. Esto significa el fortalecimiento de capacidades individuales y colectivas de todos los actores sociales implicados, a partir de caracterizar la forma en que sus prácticas comunitarias inciden en la vulnerabilidad asociada a fenómenos hidrometeorológicos agravados por el cambio climático. 


\section{CONTEXTO DE ESTUDIO}

De acuerdo con el CEPAL (2008), México se encuentra dentro de los países con altos índices de vulnerabilidad; por mencionar algunas cifras: el $71 \%$ de su PIB, el $68 \%$ de su población y el 15\% de su territorio están expuestos al riesgo climático. En este marco, el estado de Veracruz es uno de los más vulnerables; cuenta con 720 kilómetros de costa continental bañada por el Golfo de México y con una población superior a los siete millones de habitantes que enfrenta distintos eventos climáticos durante el año: sequías, lluvias torrenciales, marejadas e inundaciones, así como alteración del litoral y del relieve costero e incremento en el nivel del mar. Por estos y otros factores que no podemos mencionar aquí, el estado de Veracruz tiene un índice global de desarrollo humano (IDH) de 0.780 (datos de 2010) (PNUD, 2016) y un índice de marginación alto equivalente a un índice de 0 a cien de 57.63 ocupando el $4 .^{\circ}$ lugar nacional (CONAPO, 2010).

Debido a las características geomorfológicas de Veracruz, las agudas condiciones socioeconómicas de la mayoría de la población y su alta densidad demográfica asentada en zonas de alto riesgo, numerosas localidades con altos índices de vulnerabilidad están expuestas y sufren por ello las consecuencias de impactos de fenómenos como ciclones y tormentas tropicales. Si bien es cierto que se trata de eventos naturales, también lo es que su ocurrencia e intensidad están cada vez más relacionadas con el cambio climático e impactan la salud humana, la economía y los ecosistemas, entre otros, vulnerando así la calidad de vida de los veracruzanos.

Dicha vulnerabilidad se acrecienta ante una escasa y endeble infraestructura mermada por la corrupción e ineficiencia, así como por políticas de desarrollo desigual que mantienen en precarias condiciones de vivienda y limitados medios de subsistencia a la población más pobre periódicamente afectada por desastres ante los cuales no llega la respuesta y apoyo de las autoridades de manera equitativa y puntual antes, durante y después de los episodios extremos.

\section{DisEÑO METODOLÓGICO}

El estudio que origina este artículo se realizó con el objetivo de contribuir a fortalecer las capacidades sociales para incrementar la resiliencia de las tres comunidades implicadas en el mismo. Esto mediante la caracterización de las prácticas comunitarias y su incidencia sobre la vulnerabilidad asociada a fenómenos hidrometeorológicos agravados por el cambio climático. Lo anterior con el fin de derivar criterios y pautas de acción que, desde la educación ambiental, contribuyan a reducir los riesgos en estas y otras comunidades en condiciones equivalentes. Se dirigió a jóvenes estudiantes de nivel bachillerato como población principal, a partir del supuesto de que los jóvenes de esa edad (15-18 años) conocen las dinámicas culturales de su población de adscripción, son menos resistentes a proporcionar información para estudios de este tipo, son agentes de cambio en sus comunidades y se incorporan fácilmente a los llamados a participar en situaciones 
de emergencia ${ }^{1}$. Se aplicó una encuesta a una muestra representativa de jóvenes de los bachilleratos en las localidades de José Cardel, en el municipio de La Antigua; Cotaxtla y Tlacotalpan, en municipios del mismo nombre. Para definir la muestra, primero se realizó una selección al azar entre las escuelas de nivel medio superior (bachillerato) en los municipios donde se ubican las tres localidades de estudio, considerando que en Tlacotalpan existen tres bachilleratos, en La Antigua hay diez y en Cotaxtla se cuenta con otros tres. Se seleccionaron en total cinco bachilleratos y la muestra estuvo comprendida por 411 jóvenes (Tabla 1).

TABLA 1

NÚMERO DE PERSONAS QUE INTEGRARON LA MUESTRA EN CADA LOCALIDAD

\begin{tabular}{|c|c|c|c|c|c|}
\hline \multirow{2}{*}{ LOCALIDAD } & \multirow{2}{*}{$\begin{array}{l}\text { POBLACIÓN TOTAL } \\
\text { DE ALUMNOS EN } \\
\text { BACHILLERATOS } \\
\text { DE ESTUDIO }\end{array}$} & \multicolumn{2}{|c|}{$\begin{array}{c}\text { NÚMERO DE } \\
\text { BACHILLERATOS }\end{array}$} & \multirow{2}{*}{$\begin{array}{l}\text { MUESTRA } \\
\text { DE } \\
\text { ALUMNOS }\end{array}$} & \multirow{2}{*}{ ERROR ADMITIDO $^{2}$} \\
\hline & & MUNICIPIO & MUESTRA & & \\
\hline La Antigua & 4474 & 10 & 2 & 140 & $6.85 \%, B=0.0685$ \\
\hline Tlacotalpan & 2451 & 3 & 2 & 182 & $5.88 \%, B=0.0588$ \\
\hline Cotaxtla & 1290 & 3 & 1 & 89 & $8.4 \%, B=0.084$ \\
\hline Totales & 8215 & 16 & 5 & 411 & $3.96 \%, \mathrm{~B}=0.03965$ \\
\hline
\end{tabular}

La encuesta aplicada exploró en los jóvenes información relacionada con su conocimiento general de la situación que guarda la comunidad en que habitan (i. e. usos y costumbres frente a emergencias ambientales), los riesgos que identifican y qué posibles acciones se ponen o se han puesto en marcha para prevenirlos o adaptarse; asimismo, cómo reaccionan ante alertas tempranas frente a una contingencia y su participación en las actividades comunitarias dirigidas a reducir su vulnerabilidad, recuperarse de daños sufridos en experiencias previas vividas por ellos o por sus padres, así como su percepción sobre las debilidades y fortalezas comunitarias.

Como estrategia de triangulación metodológica, se aplicaron entrevistas semiestructuradas a personal directivo de los bachilleratos y a actores clave de la localidad (Tabla 2). Las entrevistas permitieron profundizar y complementar información relevante sobre problemas diversos de la localidad, afectaciones sufridas en la pasada inundación según las medidas preventivas y la exposición que tuvieron, así como la vulnerabilidad manifiesta y la resiliencia alcanzada.

1. Cabe mencionar que ante el incremento de la violencia derivada de la delincuencia organizada (narcotráfico, trata de personas, extorsiones, etc.) la población civil en México desconfía mucho de encuestadores en pesquisas de campo y por lo mismo no proporcionan datos fiables.

2. De acuerdo con la fórmula propuesta por MATEu y CASAL (2003). 
EDGAR JAVIER GONZÁLEZ-GAUDIANO Y ANA LUCÍA MALDONADO-GONZÁLEZ

AMENAZAS Y RIESGOS CLIMÁTICOS EN POBLACIONES VULNERABLES

TABLA 2

PARTICIPANTES EN LAS ENTREVISTAS A PROFUNDIDAD SEMIESTRUCTURADAS

\begin{tabular}{|c|c|c|c|c|}
\hline CARGO DEL PARTICIPANTE & JOSÉ CARDEL & COTAXTLA & TLACOTALPAN & TOTAL \\
\hline $\begin{array}{c}\text { Director y profesores } \\
\text { de bachillerato }\end{array}$ & 3 & 2 & 2 & 5 \\
\hline Presidente Municipal & 1 & $1^{*}$ & $1^{* *}$ & 3 \\
\hline Protección civil & 1 & 1 & 1 & 3 \\
\hline Personal Salud & 1 & 1 & & 1 \\
\hline Párroco & 6 & 5 & $1^{* * *}$ & 2 \\
\hline TOTAL & 1 & 6 & 15 \\
\hline
\end{tabular}

* El síndico en representación del presidente municipal atendió la entrevista.

*** Participaron en una misma entrevista el presidente municipal y el personal de protección civil.

**** Participó el cura párroco junto con un grupo de su comunidad parroquial de aproximadamente 25 personas.

Para su tratamiento analítico, las encuestas de los jóvenes fueron codificadas y capturadas en el paquete estadístico spss. Las entrevistas fueron grabadas y transcritas en su totalidad para realizar el análisis de contenido (Bardin, 1996) mediante fichas analíticas. La información cualitativa fue codificada y analizada según las siguientes categorías: 1) Problemas que afectan a cada localidad y con ello la vulnerabilidad y los riesgos identificados; 2) Comunicación preventiva o reactiva ante una inundación; 3) Participación y organización comunitaria; y 4) Resiliencia comunitaria, considerando las actividades realizadas para su recuperación postinundación.

\section{PRinCipales hallazgos}

Dado que los resultados de la encuesta aplicada a los jóvenes han sido reportados en otra publicación (González Gaudiano et al., 2017, en prensa), hemos priorizado en este artículo la información obtenida a partir de las entrevistas a profundidad con actores clave, y solo cuando lo hemos considerado pertinente integramos de manera descriptiva algunas cifras sobre los jóvenes de bachillerato con base en las encuestas aplicadas. La información aquí presentada es un avance del estudio y contribuye a etapas sucesivas, en las cuales se buscará definir criterios y pautas de acción que posibiliten reducir la vulnerabilidad y aumentar la resiliencia comunitaria.

Para facilitar la lectura de los resultados, hemos suprimido las claves alfanuméricas que identifican a las personas declarantes de cada localidad y hemos sintetizado las diversas opiniones sobre los asuntos de mayor interés para los entrevistados y los fines del estudio. Los resultados se presentan de manera general 
cuando existe coincidencia en las tres localidades, sólo se hace distinción en aspectos específicos a cada una de ellas cuando así se ha considerado necesario.

\subsection{Problemas que afectan a cada localidad y con ello la vulnerabilidad y los riesgos identificados}

A partir de la narrativa de un desastre como el vivido en las localidades de estudio en 2010, los actores entrevistados proporcionaron información sobre las distintas condiciones y problemas existentes que representan amenazas y riesgos en las poblaciones vulnerables.

Tal es el caso de los ríos de creciente súbita, mismos que desembocan en las cuencas de estas localidades. La inundación sufrida en 2010 fue agravada debido a que se abrieron compuertas de represas de afluentes que pertenecen a localidades vecinas, cuyas descargas afectaron seriamente las zonas bajas. Esto fue enfatizado por los entrevistados, principalmente en Tlacotalpan y Cotaxtla, donde el nivel del agua subió abruptamente por dichas descargas, así como por un tapón ocasionado por la palizada arrastrada que se atoró en un puente y a un cauce reducido por la urgente necesidad no atendida de dragar el río.

En la temporada de lluvias, los asentamientos irregulares próximos al río suelen ser afectados, con lo que se pierden animales domésticos, bienes diversos e incluso se exponen vidas humanas; esto se presenta en las tres localidades veracruzanas de estudio. Se ha reubicado a estas personas en riesgo sobre todo después de la inundación de 2010, cuando hubo recursos para hacerlo por parte del Fondo Nacional de Desastres Naturales (Fonden). Aunque han regresado a estos sitios junto al río, porque sus fuentes de ingreso están ahí. Además, rentan las propiedades que les fueron asignadas en zonas más seguras, con lo que obtienen un ingreso extra. Estas personas sostienen que lo que les sucedió es improbable que les vuelva a afectar y que, en todo caso, "que sea lo que dios quiera". Es decir, ven al cambio climático como un acto de dios frente al cual se consideran impotentes.

Las inundaciones no son identificadas como una grave amenaza por los entrevistados en Cardel y Cotaxtla. Afirman que un problema como el acontecido en 2010 no es recurrente, por lo que minimizan el riesgo y no consideran que un remanso de agua que les llegue a la rodilla sea una inundación. Están acostumbrados a las anegaciones periódicas con las crecientes del río en temporada de lluvias, por lo que algunos han adaptado sus casas elevando los pisos inferiores, colocando pequeños diques y suelen mover mobiliario a niveles más altos cuando llega a entrar el agua. Lo ocurrido con el paso del huracán Karl es visto como algo extraordinario. En Tlacotalpan se detecta un mayor reconocimiento de riesgo de inundación por parte de entrevistados. En cambio, los jóvenes tienen un registro dispar hacia una inundación en su localidad, en Cotaxtla solo el 31\% así lo admite; en Cardel, el 60\% y en Tlacotalpan, el 90\%. La identificación de las inundaciones como problema es fundamental para clarificar la disposición de poblaciones vulnerables a involucrarse en acciones de prevención. En estas localidades, la vulnerabilidad 
de las poblaciones vecinas al río continúa latente y de algún modo creciente. El temor persiste sólo cuando las lluvias se intensifican. Como desde 2010 sólo se han presentado crecientes que provocan lo que ellos denominan remansos, el riesgo subjetivo se reduce y con ello se postergan las medidas correspondientes.

Los problemas comunes que los entrevistados identifican en estas tres localidades están asociados a la economía, ya que destacan la falta de fuentes de empleo o la existencia de empleos mal remunerados, lo que deriva en migración de la población sobre todo masculina; hay pobreza e incluso pobreza extrema, por lo que también se observa desnutrición infantil, por mencionar una de sus secuelas. La mayoría de los jóvenes encuestados (90\%) no considera positivo el apoyo asistencial del Gobierno para combatir la desigualdad socioeconómica. Se señalan además problemas sociales como desintegración familiar, delincuencia juvenil, alcoholismo, adicciones e inseguridad. La falta de alumbrado público suficiente en las tres localidades puede estar contribuyendo a la inseguridad, lo cual es reconocido por los actores entrevistados y por la mayoría de los jóvenes encuestados (70\%). Se reconoce además falta de acceso a niveles superiores de educación, pues no se cuenta con universidades (sólo en Tlacotalpan hay nivel universitario con algunas carreras). Los jóvenes entonces deben migrar para estudiar o permanecen con el nivel de bachillerato, incorporándose a la fuerza de trabajo a temprana edad.

En cuanto a la salud, el calor intensifica problemas de dengue, zika y chikungunya, mismos que se han agravado y se mencionan como uno de los principales riesgos a los que está expuesta la población. Sólo el 20\% de los jóvenes encuestados ubican en niveles de eficiencia la atención e infraestructura del sistema de salud en estas localidades. La basura también aqueja a las tres localidades, toda vez que no se cuenta con un tratamiento apropiado y los tiraderos a cielo abierto en todo el estado son focos de infección y contaminación. Asociado a la disposición final de la basura, la colecta no se realiza de manera periódica y esto ocasiona que algunas personas recurran a la quema de la misma con la consecuente contaminación ambiental. Además, industrias y granjas locales contribuyen a la contaminación de mantos acuíferos por el mal manejo de sus desechos, con graves consecuencias a la salud pública. Entre los jóvenes encuestados, el 75\% reconocen la contaminación como uno de los principales riesgos en sus localidades.

En el caso particular de la localidad de José Cardel, el representante del municipio asegura que se trabaja en un relleno sanitario para resolver el problema de la basura a cielo abierto, además de que se ejecutan obras de drenaje para evitar que residuos sanitarios desemboquen directamente en el río; pero el resto de los entrevistados no menciona estas acciones. Apenas el 20\% de los jóvenes de ambos bachilleratos participantes en este estudio asignan niveles de eficiencia a la infraestructura actual de drenaje y sistema de alcantarillado en esta localidad. También mencionan una inversión pública para construir un muro de contención en la ribera del río, a la vista de toda la población, aunque algunos entrevistados lo denominan «el turrón de azúcar» porque no creen que haya sido diseñado adecuadamente. No 
sólo consideran que es difícil que pueda contener una inundación, además opinan que puede representar un riesgo mayor al incrementarse la acumulación de lodo.

Específicamente en Cotaxtla, los actores entrevistados reconocen como principales problemas que aquejan al municipio las condiciones de pobreza y pobreza extrema. La población se emplea sólo en las granjas o en el campo y la sequía no ha favorecido las cosechas. Incluso el 90\% de los jóvenes encuestados reconocen el riesgo de una probable sequía en su localidad. Dichas condiciones de pobreza mantienen a estas poblaciones en viviendas precarias y contribuyen con ello a su vulnerabilidad. En materia de salud identifican diabetes, enfisema pulmonar y problemas renales registrados desde edades tempranas, los cuales requieren de diálisis y no existe este tratamiento en la localidad; hay falta de atención médica y desabasto de medicamentos. El agua no es potable para el consumo humano, debido a la alta contaminación de la industria y de granjas porcinas y avícolas, principales fuentes de empleo (las cuales además queman una parte de sus desechos con la consecuente contaminación ambiental). Manifiestan los entrevistados que la empresa Petróleos Mexicanos (Pemex) ha vertido en el pasado sus desechos al río, aunque desconocen si estas prácticas se mantienen, el daño ya está hecho a los ecosistemas y difícilmente será remediado, pues las aguas continúan contaminadas. En este contexto, sostienen que la calidad del agua es muy mala y perjudicial para todo ser vivo: el ganado muere o aborta a sus crías, los peces también están muriendo y con ello la pesca. Se trata de una grave amenaza a la salud pública que no ha sido atendida por las autoridades competentes con el saneamiento y la aplicación de la regulación ambiental existente. Llama la atención que la mitad de los jóvenes encuestados de esta localidad ubican en niveles de eficiencia a la infraestructura de agua potable, lo que puede estar asociado a confundir la adquisición del vital líquido embotellado con las compañías distribuidoras, más que a su disposición como parte de los servicios públicos.

En el caso de Tlacotalpan, la falta de dragado del río ha incrementado su azolvamiento, ocasionando la desaparición de más de una decena de casas y una iglesia en las comunidades vecinas. Ahora también están en riesgo de desaparecer inundadas dos escuelas y otras casas. Los entrevistados además destacan el problema de contaminación por aguas servidas, ya que la planta de bombeo disponible no está enviando todos los fluidos a la laguna de oxidación y los desecha antes, con su consecuente contaminación del río y acuíferos. A esto se suman los desechos de ingenios y fábricas vecinas, ocasionando mala calidad del agua para consumo doméstico y para la pesca.

El problema de azolvamiento de los ríos es común en las tres localidades; el riesgo de inundación podría disminuir si se invirtiera en el dragado de los mismos. Esto se menciona por los actores entrevistados como una de las medidas preventivas que requiere atención especial por parte del Gobierno estatal e incluso federal, pues la inversión necesaria es grande. Los entrevistados denuncian el desvío de fondos por parte de las autoridades estatales de la administración saliente, que no emprendieron obras y campañas suficientes de protección civil. Solo el 3\% de los 
jóvenes reconocen que las autoridades han realizado obras públicas que contribuyen a disminuir riesgos ante futuros desastres, lo que coincide con la visión de los entrevistados.

En este panorama descrito por los entrevistados como bastante grave, se reconoce la precaria situación financiera a niveles estatal y municipal, derivada de la corrupción en que incurrió el Gobierno estatal anterior ${ }^{3}$ con la complicidad por omisión o comisión del Gobierno federal, ambos del mismo partido político. No hay recursos para atender el rezago social en materia educativa, de seguridad y, en estas comunidades, de salud. La ciudadanía denuncia falta de inversión, obras deficientes, defectuosas y abandono del campo. Por todo ello, las acciones gubernamentales en materia de protección civil y prevención de riesgo de desastres por potenciales eventos derivados del cambio climático no constituyen prioridades para la población.

Se cuestionó a los entrevistados para que de manera espontánea refirieran términos con los que asocian a la vulnerabilidad. Para su análisis, hemos agrupado estos términos en los tres tipos de vulnerabilidad propuestos por Anderson y Woodrow (1989), lo cual se muestra en la Tabla 3.

TABLA 3

TÉRMINOS QUE LOS ENTREVISTADOS ASOCIAN A VULNERABILIDAD

\begin{tabular}{|l|l|}
\hline \multicolumn{1}{|c|}{ TIPOS DE VULNERABILIDAD } & \multicolumn{1}{c|}{ TÉRMINOS ASOCIADOS } \\
\hline Vulnerabilidad físico-material & Riesgo, viviendas frágiles, peligro \\
\hline Vulnerabilidad social-organizacional & $\begin{array}{l}\text { Economía, pobreza, enfermedad, } \\
\text { incapacidad, debilidad, indefensión, secuestro }\end{array}$ \\
\hline Vulnerabilidad motivacional-actitudinal & $\begin{array}{l}\text { Depresión, desgracia, frustración, } \\
\text { agresión, irritación }\end{array}$ \\
\hline
\end{tabular}

Aclaramos que se trata de respuestas espontáneas al cuestionarles sobre qué palabras venían a su mente con el término «vulnerabilidad». Hubo algunos entrevistados que evitaron responder y se desviaron hacia otros temas. Aun sin haber profundizado en este ejercicio, fue posible identificar asociaciones libres significativas que consideramos que brindan pistas para próximas investigaciones, a fin de formar diferenciales semánticos que podrían estudiarse a través de métodos multivariados, tales como el análisis de conglomerados y de correspondencias y el escalamiento multidimensional (Pardo y Ruiz, 2002). La Tabla 4 permite ahora

3. En referencia al Gobierno de Javier Duarte, quien se encuentra en proceso de extradición de Guatemala, para ser enjuiciado por delitos de corrupción, entre otros. http://www.jornada.unam. $\mathrm{mx} / 2017 / 04 / 16 /$ politica/003n1pol. 
asociar los riesgos que identifican en cada localidad y el tipo de vulnerabilidad al cual estaría asociado.

TABLA 4

SÍNTESIS DE PELIGROS Y AMENAZAS TRADUCIDOS EN RIESGOS RECONOCIDOS EN CADA LOCALIDAD

\begin{tabular}{|c|c|c|c|c|}
\hline PELIGROS - AMENAZAS - RIESGOS & $\begin{array}{c}\text { TIPO DE } \\
\text { VULNERABILIDAD }\end{array}$ & CARDEL & COTAXTLA & TLACOTALPAN \\
\hline $\begin{array}{l}\text { Ríos de creciente súbita } \\
\text { afectan a localidades de } \\
\text { zonas bajas, cuando otras } \\
\text { de zonas altas deciden abrir } \\
\text { compuertas y enviar descargas, } \\
\text { sin prevenir consecuencias }\end{array}$ & \multirow{5}{*}{ Físico-Material } & $\mathrm{x}$ & $\mathrm{x}$ & $\mathrm{x}$ \\
\hline $\begin{array}{l}\text { Azolvamiento de ríos, urge } \\
\text { dragar los mismos para } \\
\text { aumentar su profundidad }\end{array}$ & & $\mathrm{x}$ & $\mathrm{x}$ & $\mathrm{x}$ \\
\hline Inundaciones & & & & $\mathrm{x}$ \\
\hline Sequías & & & $\mathrm{x}$ & \\
\hline $\begin{array}{l}\text { Viviendas precarias, } \\
\text { frágiles, vecinas al río }\end{array}$ & & $\mathrm{x}$ & $\mathrm{x}$ & $\mathrm{x}$ \\
\hline Inseguridad & \multirow{7}{*}{$\begin{array}{c}\text { Social- } \\
\text { Organizacional }\end{array}$} & $\mathrm{x}$ & $\mathrm{x}$ & $\mathrm{x}$ \\
\hline $\begin{array}{l}\text { Proliferación de enfermedades } \\
\text { derivadas de dengue, } \\
\text { zika, chikungunya }\end{array}$ & & $\mathrm{x}$ & $\mathrm{x}$ & $\mathrm{x}$ \\
\hline $\begin{array}{l}\text { Contaminación por basura } \\
\text { (quema de basura por } \\
\text { falta de colecta periódica, } \\
\text { basureros a cielo abierto) }\end{array}$ & & $\mathrm{x}$ & $\mathrm{x}$ & $\mathrm{x}$ \\
\hline $\begin{array}{l}\text { Contaminación de mantos } \\
\text { acuíferos por parte de industria, } \\
\text { granjas locales, aguas servidas }\end{array}$ & & $\mathrm{x}$ & $\mathrm{x}$ & $\mathrm{x}$ \\
\hline Pobreza y pobreza extrema & & & $\mathrm{x}$ & \\
\hline $\begin{array}{l}\text { Débil economía, falta } \\
\text { de empleos y empleos } \\
\text { mal remunerados }\end{array}$ & & $\mathrm{x}$ & $\mathrm{x}$ & $\mathrm{x}$ \\
\hline $\begin{array}{l}\text { Falta de atención médica y } \\
\text { desabasto de medicamentos }\end{array}$ & & & $\mathrm{x}$ & $\mathrm{x}$ \\
\hline
\end{tabular}




\subsection{Comunicación preventiva o reactiva ante una inundación}

Entre los entrevistados hay reconocimiento de algunos planes de prevención de riesgos de inundación que se difunden ocasionalmente en cada localidad, los que se mencionan son:

1) En José Cardel: Plan de contingencias de Protección Civil, Plan de protección interno escolar; Capacitación de paramédicos en brigadas especiales de respuesta en el bachillerato; Pláticas preventivas con el estudiantado;

2) En Cotaxtla: Jornadas de salud a cargo del Club Rotarios; simulacros por parte de Pemex;

3) En Tlacotalpan: Plan de refugios o albergues.

Por su parte, las autoridades municipales reconocen el valor del Atlas Nacional de Riesgos, aunque no se trata precisamente de un plan de prevención, sino de un diagnóstico sobre la situación de riesgo que guarda cada localidad de los municipios del país. Entre los jóvenes encuestados tampoco hay un alto reconocimiento hacia planes de prevención, solo el 18\% identifica un sistema de alerta temprana y el 13\% conoce un plan comunitario de emergencias. No existe planeación para una mejor gestión de riesgos en estas localidades. Quizás porque falta una coordinación institucional con atribuciones y competencias para su diseño y difusión entre la comunidad.

Ante una contingencia como la que se presentó en 2010, en las tres localidades hubo ausencia de comunicación oportuna a la población y a los centros escolares sobre medidas de prevención, por parte de la oficina de protección civil del estado o de alguna otra autoridad municipal. La información se presentó al mismo tiempo que la contingencia, lo cual no brindó un margen de maniobra a la población. Aunque no se ha presentado nuevamente un evento de tal magnitud, las condiciones son similares en la actualidad. Los avisos se distribuyen a través de impresos sencillos en cada domicilio o mediante perifoneo, consistente en un coche con bocinas que recorre las calles anunciando la situación y la posible necesidad de abandonar sus hogares para acudir a los albergues.

El llamado de alerta también puede llegar por parte de líderes locales que organizan a la comunidad, tal y como sucedió en 2010, cuando todos dormían y llegó la inundación por la noche junto al llamado de abandonar sus hogares para trasladarse a lugares más seguros. Debido a que éstas son medidas de reacción ante la contingencia -improvisadas y sin anticipación alguna- que no provienen de la autoridad responsable, la población se mantiene escéptica a las alertas de último momento y suele negarse a abandonar sus hogares por quedarse a proteger sus pertenencias, lo que incrementa la vulnerabilidad. La reacción tardía, desorganizada y parcial ha provocado pérdida de vidas humanas y más daños materiales. Esto coincide con el bajo reconocimiento de los jóvenes sobre la existencia de grupos de emergencia comunitaria para el manejo de desastres. Pese a esta carencia en las medidas preventivas y a la ausencia de un papel protagónico por parte de la 
autoridad responsable, la mitad de los jóvenes considera que la comunidad supo organizarse por su cuenta y enfrentar colaborativamente la contingencia en 2010.

En los planteles escolares de nivel medio superior que constituyeron la muestra para este estudio, señalaron que les gustaría contar con un plan preventivo, a fin de que directivos y profesores pudiesen transmitirlo a alumnos y padres de familia. Por ahora, la información sobre las amenazas proviene principalmente de los medios masivos: televisión, redes sociales y radio. Las medidas que frecuentemente se adoptaron por parte del Gobierno estatal saliente de suspender generalizadamente la actividad escolar frente a la amenaza de un fenómeno natural anunciado fueron extremas y a menudo innecesarias. Los directivos de los bachilleratos estudiados sugieren una mejor identificación de aquellas zonas que realmente se verán afectadas, puesto que puede registrarse una fuerte amenaza de tormenta en el sur que no implica afectar el norte del estado o viceversa.

\subsection{Participación y organización comunitaria}

Con respecto a la organización comunitaria, se detecta que ésta es más bien de tipo reactivo que preventivo. La solidaridad y apoyo emerge por parte de la misma comunidad y de agentes externos que han colaborado en la recuperación después de la contingencia. Quienes resultan menos afectados ayudan en acciones de restauración y limpieza de las viviendas de quienes han sido más afectados; la cohesión social se manifiesta ex-post, aunque no en la organización previa. La mitad de los jóvenes encuestados no identifica aún la presencia de un grupo de emergencias comunitarias capacitado en el manejo de desastres; tampoco la existencia de una red de organismos comunitarios y públicos para el apoyo ante desastres, aunque la otra mitad afirma que la comunidad ha sabido responder y actuar frente a un desastre y el 43\% reconoce que la comunidad está preparada para tomar las riendas y gestionar el proceso de su propia recuperación, después de un desastre.

A partir de la inundación de 2010 en las localidades del estudio, emergieron algunos grupos comunitarios, tales como brigadas de jóvenes de bachillerato en la localidad de José Cardel, quienes han participado en otras contingencias apoyando a la comunidad y han recibido capacitación en organización comunitaria y en medidas de primeros auxilios. También los párrocos de las iglesias católicas han organizado reuniones para estar más preparados ante contingencias. Ahora cuentan con un área para cocina colectiva mejor que la improvisada en 2010, así como impulsaron la creación de una red con otras parroquias vecinas para brindarse apoyo cuando se presenten emergencias; en esto participa también la organización católica Caritas de Veracruz.

En la localidad de Cotaxtla hay un comité ciudadano de vigilancia del río, el cual se organiza en temporada de lluvias para monitorear el aumento del nivel del río y avisar con más oportunidad a autoridades y población, de ser necesario. Los miembros de este comité ciudadano se comunican entre sí vía whatsapp, con lo que se confirma la importancia de las redes sociales para mantenerse informados. 
En Tlacotalpan existe el consejo municipal integrado por el presidente municipal y otros actores de gobierno, pero también por agentes municipales que representan a la sociedad civil, para organizar acciones preventivas y reactivas ante posibles contingencias y se contactan entre sí vía telefónica o por redes sociales.

En las tres localidades se reconoce que el personal del municipio periódicamente se reúne e informa acerca de las actividades en las que participan sobre prevención de riesgos y protección civil. A pesar de la ausencia de planes y programas bien definidos ahora están más organizados, porque en 2010 hasta las autoridades desconocían la magnitud de la inundación que enfrentarían y fue algo nuevo para todos. Sin embargo, es fundamental que esta preparación e información preventiva sea difundida entre la comunidad oportunamente, además de mantener a estas localidades equipadas con albergues, instalaciones de primer nivel de salud y centros hospitalarios, personal médico y medicamentos, así como servicio de bomberos.

Es importante, además, incrementar la interacción con los jóvenes desde los distintos espacios públicos donde se están organizando esos planes de prevención de riesgos, ya que solo entre uno y dos jóvenes encuestados considera en niveles de eficiencia su interacción con autoridades regionales y locales, organizaciones de la sociedad civil, jefe de manzana, ministro de culto, los servicios del sector salud, los servicios de protección civil y la policía municipal. Estos bajos niveles de involucramiento contribuyen a su desconocimiento e implicación en los planes de prevención que mencionan los actores entrevistados.

El liderazgo de algunos actores se ha manifestado durante las contingencias; se mencionan los siguientes: 1) en la localidad de José Cardel: el cura párroco y el director del bachillerato; 2) en Cotaxtla: los agentes municipales, los vocales de Prospera (programa social del Gobierno federal); 3) en Tlacotalpan también se menciona a los agentes municipales y los jefes de manzana. En las tres localidades se valora el liderazgo de los comisarios ejidales. Las características que mencionan en estos líderes emergentes son su aprecio y valía por parte de la comunidad, presencia durante la contingencia, solidaridad, carisma, capacidad de coordinación, gestión sociopolítica, confiabilidad y honestidad.

\subsection{Resiliencia comunitaria}

Para una pronta recuperación física y emocional de la comunidad, se reconoce la importancia de intercambiar la experiencia vivida con otros afectados; la perciben como una forma de desahogo y apoyo mutuo que se propicia durante las tareas de limpieza y restauración después de una contingencia. Ronan y Johnston (2005) explican que en estos procesos de producción y circulación de conocimiento se promueve la resiliencia social. En Cotaxtla enfatizan que en 2010 hizo falta este intercambio debido a que la distribución de víveres y de comidas preparadas por voluntarios llegaba a cada domicilio y no hubo un lugar donde se congregaran como comunidad para tener acceso al diálogo que propicia la recuperación 
colectiva. Pese a ello, en las tres localidades se reconoce que, durante la contingencia y después de ésta, disminuyó el individualismo entre los miembros de la comunidad, se incrementó la solidaridad, la comunicación y con ello la cohesión social.

En este contexto y parafraseando a Suárez Ojeda (2001) habrá que sacar algún provecho de los desastres, cuando brindan la oportunidad de analizar lo que sucede en la comunidad y es posible replantear atributos de la población, así como rescatar condiciones colaborativas para enfrentar la adversidad y buscar conjuntamente el bienestar.

Específicamente en las localidades de estudio, la gente aprovechó la ayuda que llegó por parte del Gobierno federal, de otros estados y de la sociedad civil, a la cual tuvo acceso para reconstruir al menos alrededor de una tercera parte de su patrimonio y, después de esto, continuar con su cotidianeidad para tratar de recuperar los elementos básicos de la dinámica social que se tenía antes del desastre. Sin embargo, en la memoria colectiva está registrado este desastre y el temor de que se repita algo similar, lo cual ha dejado aprendizajes que favorecen la prevención ante futuras contingencias. Ahora la comunidad posee más elementos para entender lo que sucede y atiende las indicaciones de Protección Civil, y, ante las alertas de abandonar sus hogares para acudir a resguardarse en albergues, ha habido menos resistencia. En la comunidad existe ahora mayor comunicación porque conocen los teléfonos de emergencia, además de las redes sociales informales que se han creado con este fin.

Como investigadores hemos observado que ahora están más abiertos a la presencia del cambio climático y de los daños que puede ocasionar la contaminación, también de la forma en que esto puede agravar la frecuencia e intensidad de los fenómenos naturales. Incluso cuando a los jóvenes se les cuestiona sobre la importancia de involucrarse de manera comprometida en programas y acciones para proteger el medio ambiente de manera personal, el 86\% responde afirmativamente; el $75 \%$ asegura que también su familia está participando en ello, aunque solo el $52 \%$ reconoce este incremento de participación en la comunidad en la que viven. El $70 \%$ de los jóvenes encuestados estarían dispuestos a realizar en comunidad un diagnóstico participativo para tener la información necesaria ante una emergencia. Asimismo, manifiestan el interés de participar en la organización de brigadas para capacitar a la comunidad en medidas preventivas; de diseñar planes para actuar en situaciones de emergencia, así como de asistir a asambleas comunitarias de información, sensibilización y organización preventiva. Estas disposiciones serán tomadas en consideración durante la siguiente etapa del estudio dirigida a fortalecer la resiliencia social a partir del desarrollo de medidas que favorezcan la organización y capacitación de la comunidad a través de los jóvenes implicados.

\section{CONCLUSIÓN}

Consideramos que este estudio puede ser replicado en otras comunidades en condiciones equivalentes y sus resultados pueden ser útiles para explicar situaciones 
semejantes de vulnerabilidad frente a fenómenos hidrometeorológicos extremos. De igual forma, la entusiasta participación de los jóvenes en el estudio nos ha confirmado que nuestros supuestos eran correctos, en cuanto a su disposición a colaborar y fungir como agentes de cambio en sus familias y en el resto de la comunidad. Esto a partir de ejercer liderazgo en la organización de brigadas comunitarias de protección civil que contribuyan a fortalecer la solidaridad, la cohesión social y por ende su resiliencia.

En el caso de esta investigación ha sido posible identificar y dar seguimiento a acciones permanentes de organización comunitaria, de prevención, de trabajo colaborativo para estar preparados ante próximas contingencias al haber aprendido de la anterior, así como reconocer las características que favorecen la resiliencia comunitaria y que podrían potenciarse mediante acciones educativas puntuales.

Es evidente el aprendizaje social que las localidades implicadas en este estudio han tenido como consecuencia de la experiencia previa. Esto no significa que se trate de comunidades que han dejado de ser vulnerables. Como hemos sostenido aquí, la vulnerabilidad depende de numerosos factores sociales, empezando por la ocupación particular del espacio que habitan, aledaño a cuerpos de agua y en viviendas precarias, lo cual constituye per se un factor objetivo de riesgo. Sin embargo, es válido reconocer que el riesgo subjetivo de la población ha cambiado, al otorgar ahora mayor importancia que antes a la posibilidad de ser afectada por episodios recurrentes de inundación. Si bien la población todavía considera estos fenómenos como eventos aislados, existe temor de un acontecimiento similar, por lo que la respuesta ante una alerta de evacuación es atendida de manera diligente cuando se han presentado lluvias intensas. Representar los riesgos de inundación no como sucesos ocasionales, sino como procesos de largo alcance para los que hay que estar preparados, ha de ser una lección aprendida.

De la calidad de ese aprendizaje dependerá el fortalecimiento de la resiliencia social, que les permitiría a estas localidades no sólo encarar de mejor manera las contingencias ambientales que padecen, sino desarrollar procesos de recuperación más efectivos y eficaces, sin depender tanto de la capacidad de gestión de parte de las autoridades responsables a niveles estatal y local que, como está registrado, al menos en estos casos es bastante precaria.

En ese proceso de conocer cómo se modifica ese riesgo subjetivo, lo que puede ser estratégico para impulsar programas educativos efectivos, es fundamental «entender el papel de la memoria y el olvido como un proceso de mediación entre la experiencia de desastres recurrentes y la reducción del riesgo" (Báez Ullberg, 2015, 50). Ha sido clara a lo largo del estudio realizado la presencia de una "lógica de omisión" que Báez Ullberg describe como constituida por un patrón particular de recuerdos y olvidos selectivos. Esta lógica es una parte medular del proceso mediante el cual la población va normalizando, naturalizando, el desastre recurrente de inundación, en este caso, como una característica ineludible de la cotidianeidad de sus vidas. 
Podemos afirmar que en la construcción de esa lógica selectiva de reconstrucción social sobre la experiencia del desastre de inundación sufrida en 2010 y de los episodios menores desde ese año a la fecha, las teorías ingenuas, es decir, ese "conjunto relativamente consistente de creencias que sirven para predecir un cierto número de fenómenos» (Estrada et al., 2007, 111; véase también Levy et al., 2006), han intervenido de manera sustantiva en la constitución de una específica representación social del riesgo. Es por eso que en el estudio hemos encontrado expresiones entre algunos sectores de la población que manifiestan un fatalismo religioso y resignación ante lo que ocurre.

Dicha lógica de omisión también contribuye a refrendar las incompetentes políticas de gestión del riesgo establecidas por relaciones de poder específicas, que dan prioridad a ciertos sectores de población y áreas urbanas, mientras mantienen en el abandono las zonas rurales y pequeñas poblaciones precisamente más vulnerables. De ahí que los desastres ponen de manifiesto no sólo las relaciones de poder existentes, sino también las condiciones de vulnerabilidad de una población, y sobre todo las formas culturales que inciden en la definición de lo que es riesgoso y lo que no es tal. Hemos confirmado en otros estudios (González-Gaudiano y Maldonado, 2013, 2014; Maldonado y González-Gaudiano, 2013; González-Gaudiano, 2012; González-Gaudiano y Meira, 2009), que la representación social del cambio climático determina la forma en la que las personas de estas comunidades perciben su propio riesgo y vulnerabilidad y en consecuencia el comportamiento de este grupo social frente a contingencias extremas, derivadas o no de este fenómeno global.

El riesgo es una representación, por eso es subjetivo (Douglas, 1996) y, al incidir en la concepción de una dimensión de la realidad como la relacionada con la vulnerabilidad ante inundaciones, define también la disposición individual y social para poner en marcha ciertas estrategias, dispositivos y prácticas dirigidas a mitigar los impactos de la adversidad en la vida de las personas. Un propósito muy claro de estrategias educativas dirigidas a fortalecer esa disposición a actuar tiene que consistir en expurgar de la representación social tanto los fatalismos como la desesperanza, la incertidumbre y la negación de fenómenos climáticos que ya forman parte de nuestras vidas, aunque no los queramos en ellas. Esto es, construir otra lógica selectiva de la memoria, otra "forma de ver» (De Certeau, 1996) donde destaquen los esfuerzos colectivos para realmente tomar el dominio de nuestras vidas, para resignificar los problemas que enfrentamos y derivar hacia un aprendizaje social de mejor calidad sobre las experiencias que vivimos por catastróficas que parezcan.

\section{REFERENCIAS BIBLIOGRÁFICAS}

Adger, N. (1999) Vulnerabilidad Social al Cambio Climático y Extremos en el Costero de Vietnam. World Development, 27 (2), 249-269.

ANDERson, M. y WoOdrow, P. (1989) Rising from the Ashes. Development Strategies in Times of Disaster. Boulder (Colorado), Westview Press-unEsco. 
Ávila Flores, B. y González-Gaudiano, E. (2014) Percepción social de los eventos climáticos extremos: una revisión teórica enfocada en la reducción del riesgo. Revista Trayectorias, 16 (39), 36-58.

Báez Ullberg, S. (2015) La gestión de las inundaciones y la lógica de la omisión en la ciudad de Santa Fe, en Viand, J. y Briones, F. (comps.) Riesgos al sur. Diversidad de riesgos de desastres en Argentina. Buenos Aires. La Red-Imago Mundi, 49-60.

Bardin, L. (1996) Análisis de contenido. Madrid, Ediciones Akal.

BECK, U. (2000) Retorno a la teoría de la sociedad del riesgo. Boletín de la AGE, 30, 9-20.

BECK, U. (2008) La sociedad del riesgo mundial. En busca de la seguridad perdida. Barcelona, Editorial Paidós Estado y Sociedad.

Brachin, S. (2003) Comparative public opinion and knowledge on global climatic change and the Kyoto Protocol: The U.S. versus the World? International Journal of Sociology and Social Policy, 23 (10), 106-134.

BRIONES, F. (2010) Inundados, reubicados y olvidados: Traslado del riesgo de desastres en Motozintla, Chiapas. Revista de Ingeniería, 31, 132-144.

CEPal (2008) Amuario Estadístico de América Latina y el Caribe. Organización de las Naciones Unidas.

CONAPO (2010) Estimaciones del CONAPO con base en el INEGI. Censo de Población y Vivienda 2010. México, CONAPO.

De Certeau, M. (1996) La invención de lo cotidiano, vol. 1. México, DF, Universidad Iberoamericana.

Douglas, M. (1996) La aceptabilidad del riesgo según las ciencias sociales. Barcelona, Paidós.

DunlaP, R. E. (1998) Lay perceptions of global risk: Public views of global warming in crossnational context. International Sociology, 13 (4), 473-498.

Estrada, C.; Oyarzún, M. e Yzerbyt, V. (2007) Teorías implícitas y esencialismo psicológico: Herramientas conceptuales para el estudio de las relaciones entre y dentro de los grupos. Psykhe, 16 (1), 111-121.

Fernández, P.; Mourato, S.; Moreira, M. y Pereira, L. (2016) Spatial flood vulnerability assessment. Decision makers' challenge. Helsinki, AGILE.

García Acosta, V. (2005) El riesgo como construcción social y la construcción social del riesgo. Desacatos, 19, 11-24.

GONZÁLEZ-GAUDIANO, E. (2012) La representación social del cambio climático: Una revisión internacional. Revista Mexicana de Investigación Educativa. México, comie, 17 (55), 1035-1062.

GonZÁlez-Gaudiano, É. J. y MALdONAdo GonZÁlez, A. L. (2013) Los jóvenes universitarios y el cambio climático. Un estudio de representaciones sociales. Xalapa, Universidad Veracruzana. Colección Biblioteca.

González-Gaudiano, E. J. y Maldonado González, A. L. (2014) ¿Qué piensan, dicen y hacen los jóvenes universitarios sobre el cambio climático? Un estudio de representaciones sociales. Educar em Revista, 3, 35-55.

Gonzále-Gaudiano, E. J.; Maldonado González, A. L. y Cruz Sánchez, G. E. (2017) (en prensa) La visión de los jóvenes de bachillerato a su vulnerabilidad y resiliencia social frente a los embates del cambio climático en municipios de alto riesgo a inundaciones. Psyecology.

González-Gaudiano, E. y Meira Cartea, P. A. (2009) Educación, Comunicación y Cambio climático. Resistencias para la acción social responsable. Trayectorias. Revista de 
Ciencias Sociales, 11 (29), 6-38. También publicado con el título Educación y comunicación para el cambio climático. Un punto de vista crítico de los obstáculos y resistencias. Culture de la Sostenibilitá, 5, 48-74.

Halbwachs, M. (2004) La memoria colectiva. Zaragoza, Prensas Universitarias de Zaragoza. IPCC (2014) Cambio climático 2014: Impactos, adaptación y vulnerabilidad. Grupo Intergubernamental de Expertos sobre el Cambio Climático.

Levy, S. R.; Chi-Yue, C. y Ying-Yi, H. (2006) Lay theories ant intergroup relations. Group Processes and Intergroup Relations, 9 (1), 5-24.

Maldonado GonZÁlez, A. L. y GonZalez-Gaudiano, E. J. (2013) De la resiliencia comunitaria a la ciudadanía ambiental. El caso de tres localidades en Veracruz, México. Revista Integra Educativa, 6 (3), 13-28.

Mateu, E, y Casal, J. (2003) Tamaño de la Muestra, CReSA. Centre de Recerca en Sanitat Animal/Dep. Sanitat i Anatomia Animals. Barcelona, Universitat Autònoma de Barcelona, 8-10.

Moore, J. W. (2011) Transcending the Metabolic Rift: A Theory of Crises in the Capitalist World Ecology. Journal of Peasant Studies, 38 (1), 1-46.

Neri, C. y Aldunce, P. (2008) Métodos y conceptos para el estudio de la variabilidad y cambio climático, en Aldunce, P.; Neri, C. y Szlafsztein, C. (eds.) Hacia la evaluación de prácticas de adaptación ante la variabilidad y el cambio climático. Belem, NUMA/ UFPA, 11-20.

Nisbet, M. y Myers, T. (2007) The Polls-Trends: Twenty years of public opinion about global warming. Public Opinion Quarterly, 71 (3), 444-470.

NorgaARD, K. y Rudy, A. (2008) Footnotes: Newsletter of the American Sociological Association.

PARDO, A. y RuIZ, M. A. (2002) SPSS 11. Guía para el análisis de datos. Madrid, McGraw Hill.

PNUD (2016) Índice de Desarrollo Humano. México 2016. Desigualdad y Movilidad. México, Programa de Naciones Unidas para el Desarrollo.

RONAN, K. R. y Johnston, D. M. (2005) Promoting community resilience in disaster: the role for schools, youth and families. USA, Springer.

SAUVÉ, L. (2010) Educación científica y educación ambiental: un cruce fecundo. Enseñanza de las Ciencias, 28 (1), 5-18.

Sú́rez OJEDA, E. N. (2001) Una concepción latinoamericana: la resiliencia comunitaria, en Melillo, A. y SuÁrez OJedA, E. N. (comps.) Resiliencia, descubriendo las propias fortalezas. Buenos Aires, Paidós, 67-82.

ViAND, J. y GONZÁlEZ, S. (2012) Crear riesgo, ocultar riesgo: gestión de inundaciones y política urbana en dos ciudades argentinas, en Primer Encuentro de Investigadores en Formación de Recursos Hídricos. Buenos Aires, Instituto Nacional del Agua.

Wilches-Chaux, G. (1993) Vulnerabilidad Global, en Maskrey, A. (comp.) Los desastres no son naturales. Panamá, Red de estudios Sociales en Prevención de Desastres en América Latina (La Red), 11-44. 